\title{
Hamiltonian distributed chaos and predictability in large-scale climate dynamics
}

\author{
A. Bershadskii \\ ICAR, P.O. Box 31155, Jerusalem 91000, Israel
}

\begin{abstract}
It is shown that the large-scale climate dynamics, represented by the daily indices of the North Atlantic (NAO), Pacific/North American (PNA), Arctic (AO) and Antarctic (AAO) oscillations, Asian-Australian Monsoons (ISM, WNPM and AUSM) and El Niño/La Niña phenomenon (Nino indices) as well as global temperature anomalies (land), is dominated by the Hamiltonian distributed chaos with the stretched exponential spectrum. The predictability problem (predictability horizon properties) has been also discussed in this context.
\end{abstract}

\section{INTRODUCTION}

Modern approach to the deterministic chaos was initiated by the seminal paper Ref. [1]. The Lorenz system

$$
\frac{d x}{d t}=\sigma(y-x), \quad \frac{d y}{d t}=r x-y-x z, \quad \frac{d z}{d t}=x y-b z
$$

is a very simplified model for Rayleigh-Benard (thermal) convection in a layer of fluid, cooled from above and heated from below (a typical situation for atmospheric convection). The parameters $b=8 / 3, r=28.0, \sigma=10.0$ provide a chaotic solution. Fig. 1 shows power spectrum of $z$-component (in the semi-logarithmic scales). The spectrum was computed using the maximum entropy method, which provides an optimal resolution for comparatively short data sets [2]. The dashed straight line corresponds to the exponential spectrum

$$
E(f) \propto \exp -\left(f / f_{c}\right)
$$

where $f_{c}=$ const is some characteristic frequency. Many dynamical systems with chaotic behaviour (including some atmospheric phenomena) have the exponentially

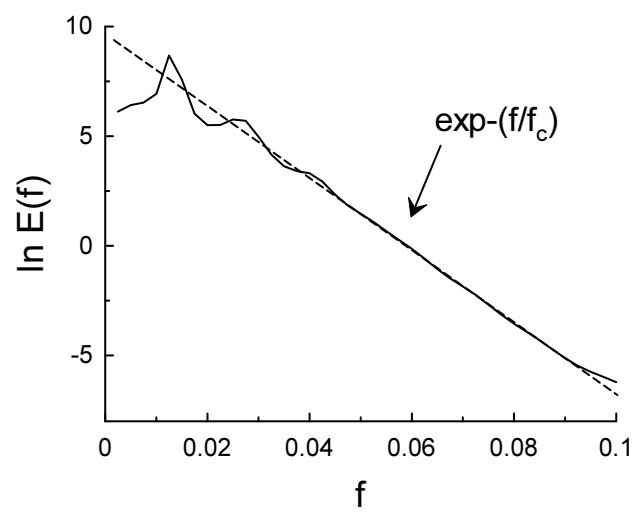

FIG. 1: Logarithm of power spectrum for $z$-component of the Eq. (1) against frequency $f$. The dashed straight line corresponds to Eq. (2). decaying power spectra Eq. (2) [2]-[6]. This can be related to the smooth sensitivity to the initial conditions mentioned by E. N. Lorenz in his 1969 paper [7]:

"The error eventually becomes much larger than the initial error. At any particular future time the error may be made arbitrarily small by making the initial error sufficiently small, but, no matter how small the initial error (if not zero), the error becomes large in the sufficiently distant future."

The initial error is a measure of the differences in the initial conditions. If the above mentioned smoothness is extended on derivatives of all orders (a $C^{\infty}$ class), then decaying part of power spectrum has a stretched exponential form [8]:

$$
E(f) \propto \int_{0}^{\infty} P\left(f_{c}\right) \exp -\left(f / f_{c}\right) d f_{c} \propto \exp -\left(f / f_{0}\right)^{\beta}
$$

where $P\left(f_{c}\right)$ is a weight (distribution) of the exponentials with the characteristic frequency $f_{c}$, and $\beta \leq 1$ (though, for the $\beta=1$ a stronger - analytical, smoothness should be demanded and the distribution $P\left(f_{c}\right)$ can be approximated by a delta-function). This is in particular true for the Hamiltonian systems (the smoothness is a necessary but not enough condition). Since most of the important theoretical models in geophysical fluid dynamics are Hamiltonian [9]-[12] the stretched exponential spectrum Eq. (3) (with certain specific values of the parameter $\beta$, see next Section) will be the main working tool in present paper.

It will be shown that dynamics of the daily indices corresponding to the main large-scale climate oscillations/patterns was under strong influence of the Hamiltonian distributed chaos with the stretched exponential power spectrum Eq. (3). Among such large-scale climate oscillations/patterns are the North Atlantic Oscillation (NAO), Pacific/North American pattern (PNA), Arctic and Antarctic Oscillations (AO and AAO), Asian-Australian Monsoons (ISM, WNPM and AUSM) and El Niño/La Niña phenomenon (Nino indices) as well as global temperature anomalies (land).

The smoothness of the chaotic behaviour is naturally related to the predictability problem (predictability hori- 


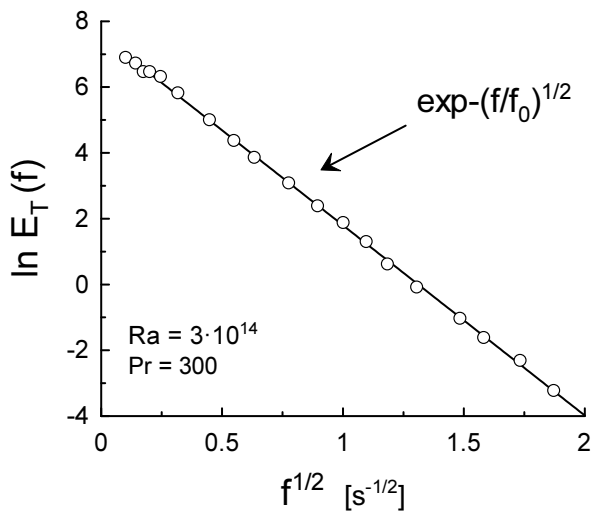

FIG. 2: Power spectrum of temperature measured at the cell center (the data taken from the Ref. [15]). The solid straight line indicates the stretched exponential decay Eq. (3).

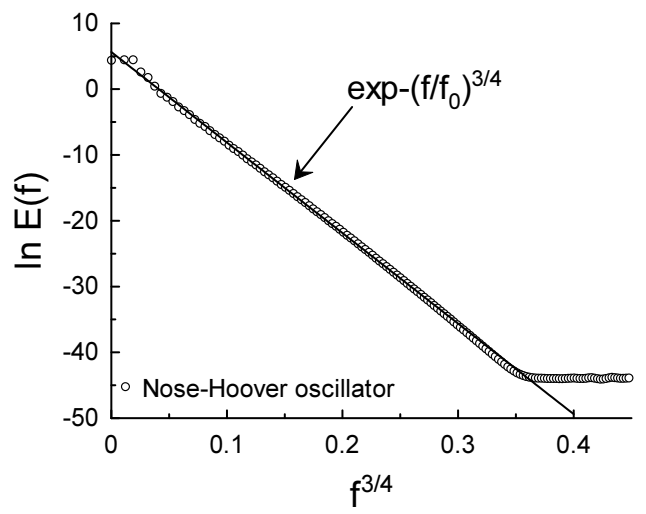

FIG. 3: Power spectrum for the Nose-Hoover oscillator. The data for the computations were taken from Ref. [20]).

zon properties). Therefore, the appearance and dominance of the stretched exponential power spectrum Eq. (3) for the most significant large-scale climate daily indices will be also discussed from this point of view.

\section{DISTRIBUTED CHAOS IN THE HAMILTONIAN DYNAMICAL SYSTEMS}

A Hamiltonian dynamical system can be described by a Hamiltonian scalar function - $H(q, p, t)$ of the two vectors - position $q$ and momentum $p$, and of the time $t$. The variables are related by the evolution equation

$$
\frac{d q}{d t}=\frac{\partial H}{\partial p}, \quad \frac{d p}{d t}=-\frac{\partial H}{\partial q}
$$

Let us expand analytically at frequency $f=0$ the power spectrum of the position $q(t)$

$$
E_{q}(f)=I_{q}+I_{q}^{(1)} f+I_{q}^{(2)} f^{2}+\ldots \ldots
$$

and the power spectrum of the momentum $p(t)$

$$
E_{p}(f)=I_{p}+I_{p}^{(1)} f+I_{p}^{(2)} f^{2}+\ldots \ldots
$$

For a time independent Hamiltonian or for the situations when the action $I$ is an adiabatic invariant [13]

$$
I_{p}=c \cdot I
$$

where $c$ is a dimensionless constant. Therefore $I_{p}$ can be considered as a momentum-action and, analogously, $I_{q}$ can be considered as a position-action.

A relationship between characteristic velocity $v_{c}$ and the characteristic frequency $f_{c}$ can be found using the dimensional considerations [13]:

$$
v_{c} \propto I^{1 / 2} f_{c}^{1 / 2}
$$

or (using Eq. (8))

$$
v_{c} \propto I_{p}^{1 / 2} f_{c}^{1 / 2}
$$

Analogously, depending on initial and boundary conditions,

$$
v_{c} \propto I_{q}^{1 / 2} f_{c}^{3 / 2}
$$

In a more general form

$$
v_{c} \propto f_{c}^{\alpha}
$$

where $\alpha$ can take the two values $\alpha=1 / 2$ or $\alpha=3 / 2$.

If probability distribution of the $v_{c}-\mathcal{P}\left(v_{c}\right)$, is Gaussian (with zero mean) then in the case of relationship Eq. (10) the variable $f_{c}$ has the chi-squared $\left(\chi^{2}\right)$ distribution:

$$
P\left(f_{c}\right) \propto f_{c}^{-1 / 2} \exp -\left(f_{c} / 4 f_{0}\right)
$$

here $f_{0}$ is a constant.

Substituting the Eq. (13) into the Eq. (3) one obtains

$$
E(k) \propto \exp -\left(f / f_{0}\right)^{1 / 2}
$$

i.e. a stretched exponential spectrum with $\beta=1 / 2$.

For the stretched exponential spectrum Eq. (3) the $P\left(f_{c}\right)$ asymptote at $f_{c} \rightarrow \infty$ is

$$
P\left(f_{c}\right) \propto f_{c}^{-1+\beta /[2(1-\beta)]} \exp \left(-b f_{c}^{\beta /(1-\beta)}\right)
$$

where $b$ is a constant [14].

The probability distributions of the characteristic velocity $\mathcal{P}\left(v_{c}\right)$ and the characteristic frequency $P\left(f_{c}\right)$ are related as

$$
\mathcal{P}\left(v_{c}\right) d v_{c} \propto P\left(f_{c}\right) d f_{c}
$$


or, taking into account the Eq. (12),

$$
P\left(f_{c}\right) \propto f_{c}^{\alpha-1} \mathcal{P}\left(v_{c}\left(f_{c}\right)\right)
$$

From the Eqs. (12),(15) and (17) one can conclude that for the Gaussian distribution of the characteristic velocity - $\mathcal{P}\left(v_{c}\right)$, the parameter $\beta$ is related to the parameter $\alpha$ by the equation

$$
\beta=\frac{2 \alpha}{1+2 \alpha}
$$

It follows from the Eq. (18) that for $\alpha=3 / 2$ (the Eq. (11)) the parameter $\beta=3 / 4$, and for $\alpha=1 / 2$ (the Eq. (10)) the parameter $\beta=1 / 2$, depending on the initial and boundary conditions.

Figure 2 shows, as an example, a power spectrum for the temperature fluctuations measured at the cell center in a laboratory thermal (Rayleigh-Benard) convection at large Rayleigh number $R a=3 \cdot 10^{14}$ (the spectral data were taken from the Ref. [15]). The Hamiltonian models of the Rayleigh-Benard convection can be found in the Refs. [16],[17]. The straight line in the Fig. 2 indicates (in the appropriately chosen scales) correspondence to the Eq. (3) with $\beta=1 / 2$. Similar spectrum was observed for the first time in experiment reported in Ref. [18].

Another relevant example is shown in Fig. 3. It is a power spectrum for the $x(t)$ variable of the Nose-Hoover oscillator. This oscillator can be considered as a harmonic oscillator contacting with a thermal bath. It is a Hamiltonian system [19]. The data used for computation of the spectrum were taken from the site [20]. The maximum entropy method with an optimal resolution [2] has been used for the computation. The straight line indicates correspondence to the Eq. (3) with $\beta=3 / 4$.

\section{NORTH ATLANTIC OSCILLATION}

In the Northern Hemisphere much of the Atlantic ocean is covered by the North Atlantic Oscillation (NAO). The NAO index is usually defined as the difference in normalized sea-level pressure anomalies between Southwest Iceland (a northern node) and Azores (a southern node). It is so-called station-based method of the NAO index computation (see, for instance, Refs. $[21],[22],[23]$ and references therein). There is also another method of the NAO index computation. This method uses gridded climate datasets with empirical orthogonal analysis - EOF (see, for instance, Refs. $[24],[25],[26]$ and references therein). There are also different modifications of the above mention methods and, therefore, there are different versions of the NAO index

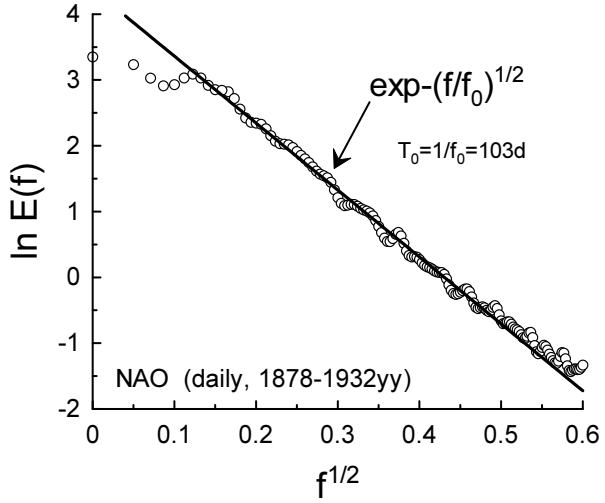

FIG. 4: Power spectrum of the NAO index computed for the period 1878-1932yy. The data for the computations were taken from the site [27].

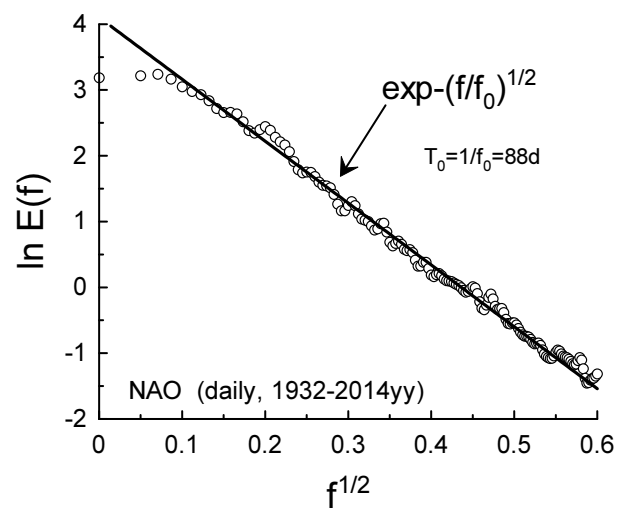

FIG. 5: As in the Fig. 4 but for the period 1932-2014yy.

(this is also right for other climate indices). All these methods have their strong and weak sides. In this paper the daily NAO index computed by the station-based method - the difference in normalized sea-level pressure anomalies between Southwest Iceland and Azores [23], has been analysed (an example of the second method will be shown in the next Section).

Figure 4 shows power spectrum of the NAO daily index computed for the period 1878-1932yy. The data for the computations were taken from the site [27]. The maximum entropy method with an optimal resolution [2] has been used for the computation. The straight line indicates correspondence to the Eq. (3) with $\beta=1 / 2$. Figure 5 shows analogous spectrum for the period 1932-2014yy.

The positive phase of the NAO corresponds to below normal pressure and heights across the high latitudes of the North Atlantic and above normal pressure and heights 
over the central North Atlantic, the western Europe and eastern United States. The negative phase corresponds to an opposite pattern of pressure and height anomalies.

Strong influence of the North Atlantic oscillation was registered in the surface temperature dynamics from eastern North America to central Europe (and even to the north-central Siberia), and from Greenland and Scandinavia to the Middle East [28] (cf. last Section).

\section{PACIFIC/NORTH AMERICAN PATTERN}

The Pacific/North American (PNA) index reflects a planetary-scale recurring pattern (atmospheric oscillations) of circulation and pressure anomalies over the $\mathrm{Pa}$ cific ocean and North America. The index is based on the quadrupole centers of action: [(15-25N, 180-140W)-(40$50 \mathrm{~N}, 180-140 \mathrm{~W})+(45-60 \mathrm{~N}, 125 \mathrm{~W}-105 \mathrm{~W})-(25-35 \mathrm{~N}, 90 \mathrm{~W}-$ 70W)] (cf. Fig. 6) [29], of 500mb height oscillating patterns (see Ref. [30] for the details of the NCEP-NCAR R1 reanalysis). It includes land (the intermountain region of North America) as one of its centers while the other three centers of the PNA pattern are ocean based (see Fig. 6). At the computations of the PNA index the area-weighted mean 500-hPa geopotential height fields of the region (40$50 \mathrm{~N}, 180-140 \mathrm{~W})$ is subtracted from the $(15-25 \mathrm{~N}, 180-$ $140 \mathrm{~W}$ ) area averaged region (the first dipole), and the area-weighted mean $500-\mathrm{hPa}$ geopotential height fields of the region $(25-35 \mathrm{~N}, 90 \mathrm{~W}-70 \mathrm{~W})$ is subtracted from the $(45-60 \mathrm{~N}, 125 \mathrm{~W}-105 \mathrm{~W})$ area averaged region (the second dipole). Then summation of the results of these subtractions (dipoles) provides the quadrupole PNA index. To emphasize large-scale properties of the PNA pattern the height fields were spectrally truncated at the index computation (see [29] for more technical details).

Figure 7 shows power spectrum of the daily PNA index (the data were taken from the Ref. [29]). The straight line indicates correspondence to the Eq. (3) with $\beta=$ $3 / 4$. The characteristic time scale inferred from the best fit is $T_{0}=1 / f_{0} \simeq 41 \mathrm{~d}$.

It should be noted that the near 40-day oscillations are well known for the North Hemisphere extratropics and are usually associated with the interaction of the non-zonal flows with topography (see, for instance, Refs. [31]-[35] and references therein).

\section{SLP DYNAMICS OF THE ARCTIC AND ANTARCTIC OSCILLATION}

The Arctic Oscillation (AO index) and the Antarctic Oscillation (AAO index) are primary annular modes of extratropical atmospheric circulation in the Northern and Southern Hemispheres respectively [25] (see for a comprehensive review Ref. [36] and references therein). In a certain sense these patterns represent a measure of
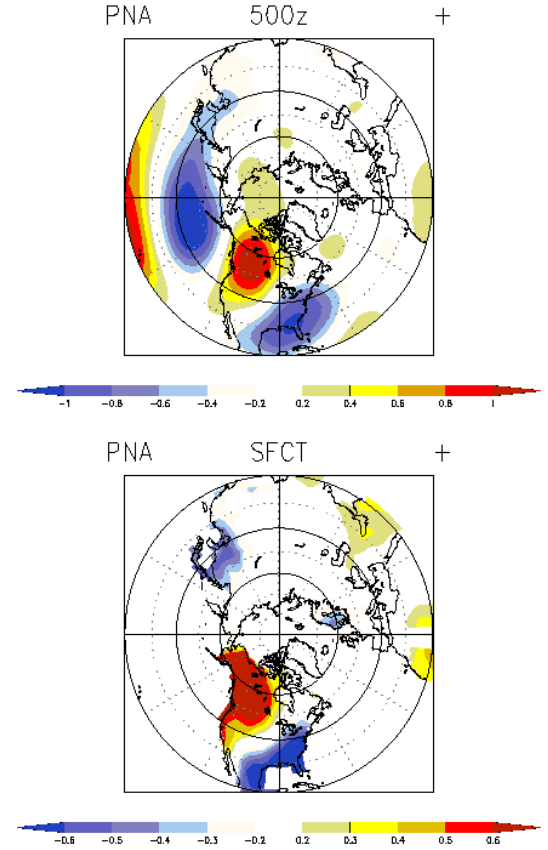

FIG. 6: Map of the PNA at 500mb height (positive phase), and corresponding surface temperature anomaly over land (the lower picture) [29].

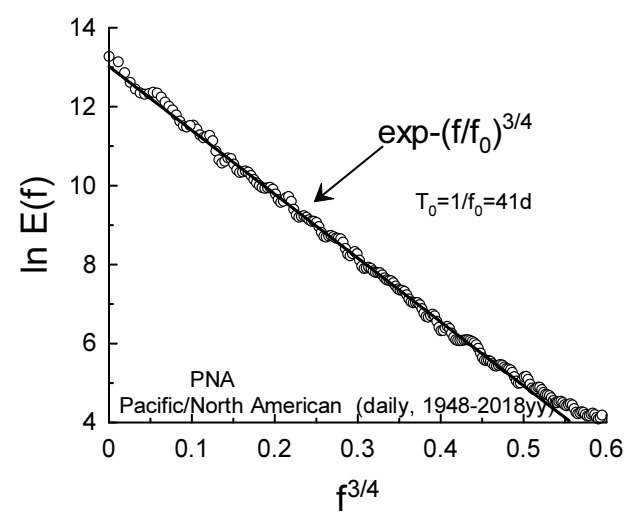

FIG. 7: Power spectrum of the PNA daily index for the period 1948-2018yy. The data for computation were taken from the site [29].

the pressure gradient between the subpolar and polar regions.

The AO and AAO are characterized by winds circulating around the Arctic and Antarctic. When the AO and $\mathrm{AAO}$ indices are in their positive phase (corresponding to low polar pressure), these strong winds confine colder air in the corresponding polar regions. When the indices are in their negative phase the ring of winds becomes 


\section{CR V2 AO Based on 1981 to 2010 SLP}
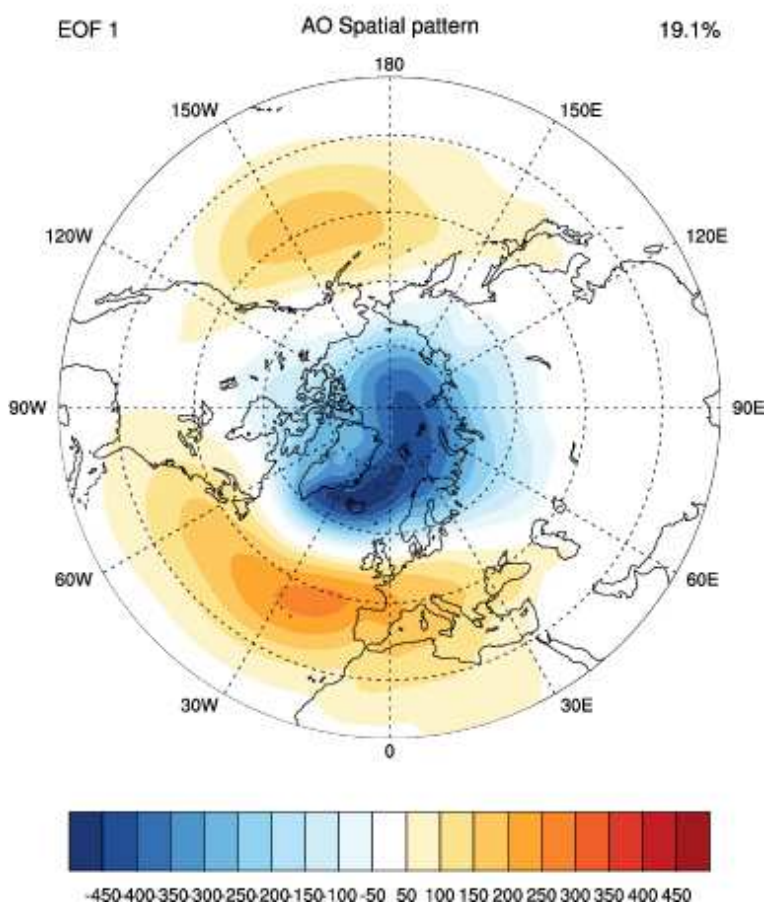

FIG. 8: Spatial pattern of the Arctic Oscillation [39].

weaker, that allows more incursions of Arctic/Antarctic colder airmasses into the subpolar regions and even into the mid-latitudes. The annular modes exist in the troposphere at all seasons. The annular pattern is a nearly zonally symmetric in the middle stratosphere corresponding to the stratospheric polar vortex [37]. It is believed that AO and AAO play a very important role in the climate variability on scales of seasons and longer.

In the seminal Ref. [25] the AO was defined as the leading principal component of $850 \mathrm{mb}$ geopotential height (elevation $1458 \mathrm{~m}$ ) anomalies poleward of $20^{\circ} \mathrm{N}$. The AAO is defind analogously with replacement of the $20^{\circ} \mathrm{N}$ by the $20^{\circ} \mathrm{S}$. At the site Ref. [38] the AO daily index was calculated at the $1000 \mathrm{mb}$ height (elevation $111 \mathrm{~m}$ ) for period 1950-2018yy and AAO daily index was calculated at the $700 \mathrm{mb}$ height (elevation $3013 \mathrm{~m}$ ) for period 1979-2018yy.

At the sites [39] and [40] the sea-level pressure (SLP) based AO and AAO daily indices were calculated for a prolonged time period. The SLP is the atmospheric pressure at sea level at a given location. If a station is not located at sea level the SLP is a correction of the station pressure to corresponding sea level (taking into account the variation of pressure with height and influence of temperature).

Figure 8, for instance, shows a spatial pattern of the Arctic Oscillation. The Empirical Orthogonal Function

\section{CR V2 AAO Based on 1981 to 2010 SLP}

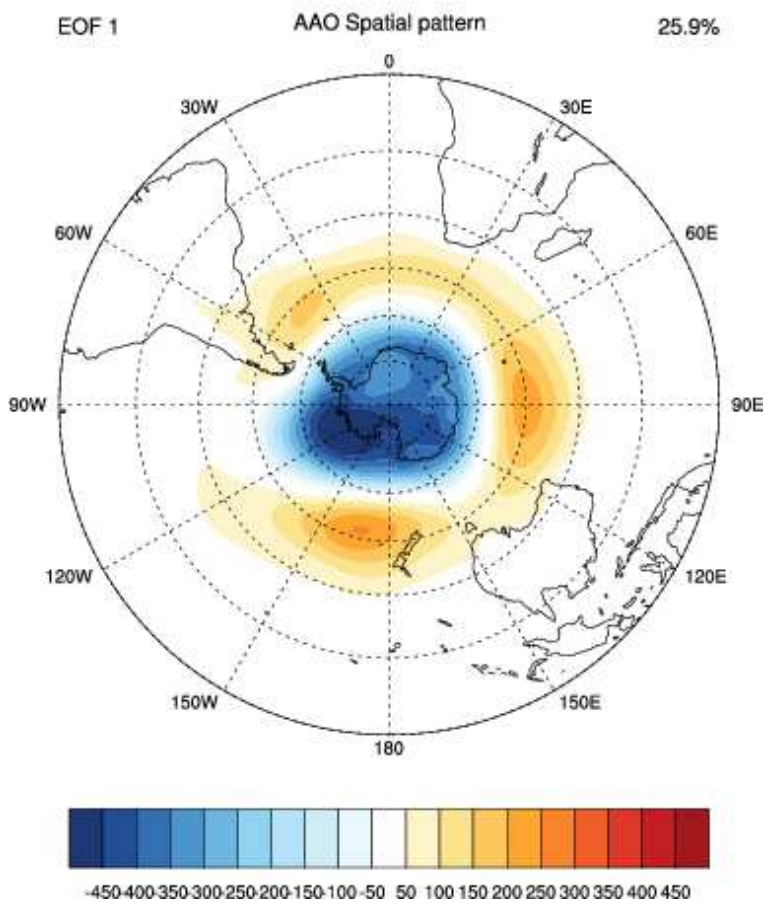

FIG. 9: Spatial pattern of the Antarctic Oscillation [40].

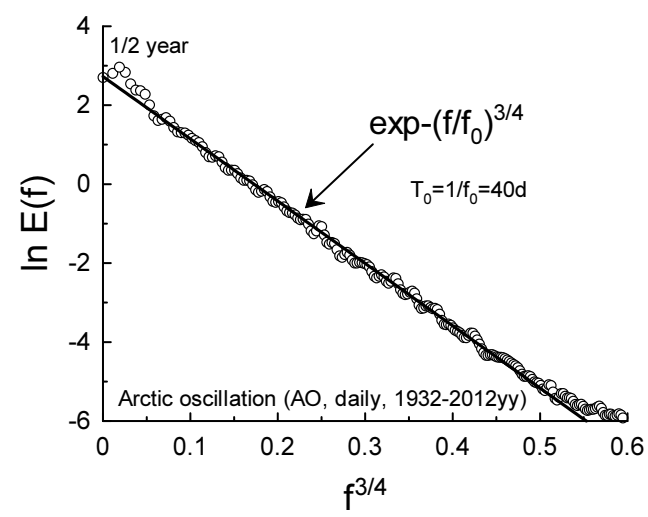

FIG. 10: Power spectrum of the AO daily index for the period 1932-2012yy. The data for computation were taken from the site [39].

(EOF) is based on 1981-2010yy monthly anomalies of SLP poleward of $20^{\circ} \mathrm{N}$ (the 20CR V2 dataset) [39]. The AO daily index was obtained by projecting the daily anomalies of SLP from 1871-2012 onto the EOF's. The Antarctic Oscilation (AO) daily index has been constructed analogously with replacement of the $20^{\circ} \mathrm{N}$ by the $20^{\circ} \mathrm{S}$ (see Fig. 9) [40]. 


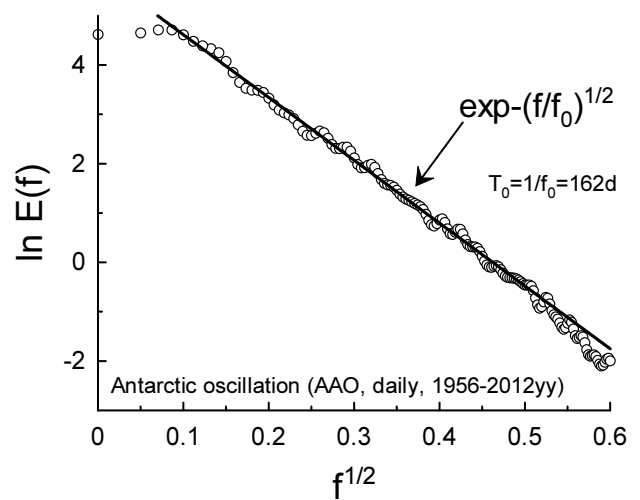

FIG. 11: Power spectrum of the AAO daily index for the period 1956-2012yy. The data for computation were taken from the site [40].

Figure 10 shows power spectrum computed for the daily AO index for period 1932-2012yy (the timeseries were taken for the site [39]). The maximum entropy method with an optimal resolution [2] has been used for the spectra computation. The straight line indicates correspondence to the Eq. (3) with $\beta=3 / 4$. The characteristic time scale inferred from the best fit is $T_{0}=1 / f_{0} \simeq 40 \mathrm{~d}$ (cf. Fig. 7 for the PNA daily index: one can see a strong spectral affinity between the PNA and AO daily indices). Figure 11 shows power spectrum computed for the daily AAO index for period 19562012yy (the timeseries were taken for the site [40]). The straight line indicates correspondence to the Eq. (3) with $\beta=1 / 2$. The characteristic time scale inferred from the best fit is $T_{0}=1 / f_{0} \simeq 162 \mathrm{~d}$.

\section{THE ASIAN-AUSTRALIAN MONSOONS AND EL NIÑO/LA NIÑA PHENOMENON}

\section{Asian Monsoons}

Two dominant regions of energy supply (by convective heat) can be determined for the Asian monsoon: the Philippine Sea and Bay of Bengal (see for a comprehensive review Ref. [41]). Weak correlation between these rather different energy sources resulted in appearance of two different in their properties monsoon indices: Western North Pacific Monsoon Index (WNPMI) and Indian Summer Monsoon Index (ISMI or IMI) [41],[42]. Together these indices describe one of the most powerful oscillating pattern of the earth's climate. Definition of ISMI and WNPMI can be understood from the figure 12 [41],[43]. The regions where the zonal winds are used for computation of the monsoon circulation indices are denoted in the Fig. 12 by the boxes.

According to the Refs. [41],[42] the difference of the
Asian Summer Monsoon Indices

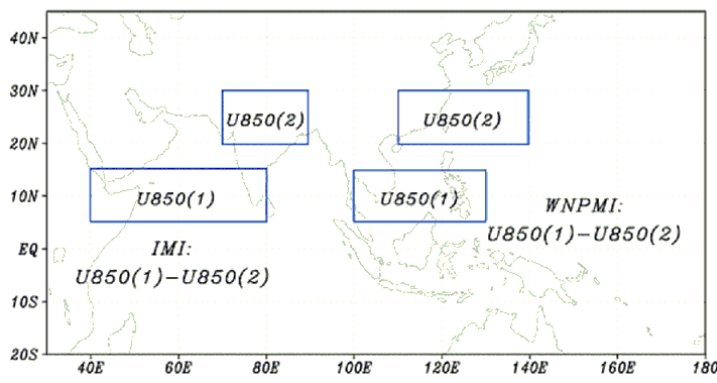

FIG. 12: Map for the Indian Summer Monsoon Index (left) and Western North Pacific Monsoon Index (right) [43].

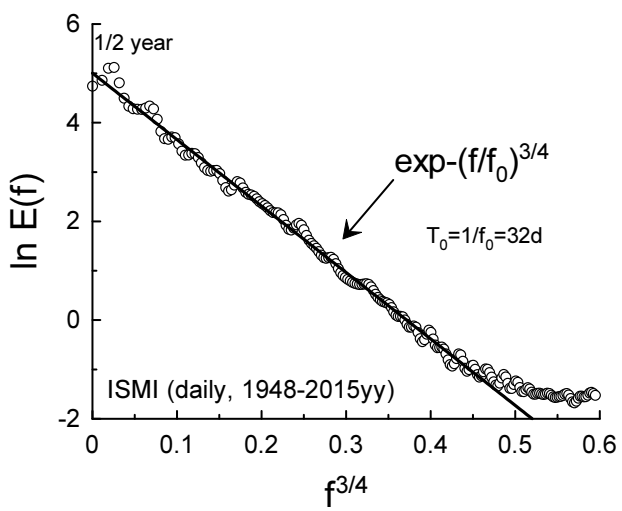

FIG. 13: Power spectrum for the intraseasonal daily ISM index for the period 1948-2015yy (the data for the computation were taken from the site [43]).

$850-\mathrm{hPa}$ zonal winds is used for the definition of the

$$
I S M I=U 850(40 E-80 E, 5 N-15 N)-U 850(70 E-90 E, 20 N-30 N)
$$

and the difference of $850-\mathrm{hPa}$ westerlies is used for the definition of the

$W N P M I=U 850(100 E-130 E, 5 N-15 N)-U 850(110 E-140 E, 20 N-30 N)$

The ISMI represents the rainfall anomalies over a region including the India, Bay of Bengal and the eastern Arabian Sea. The WNPMI represents also the low-level vorticity generated by response of the Rossby waves to convective heat source located at the Philippine Sea (cf. the Ref. [44]). Correlation between these indices is very weak that corresponds well to the difference in their origins. Dissimilarity between the ISM and WNPM geographic setting in respect of the ocean-continent distribution results in the considerable differences in their variability for all time scales. 


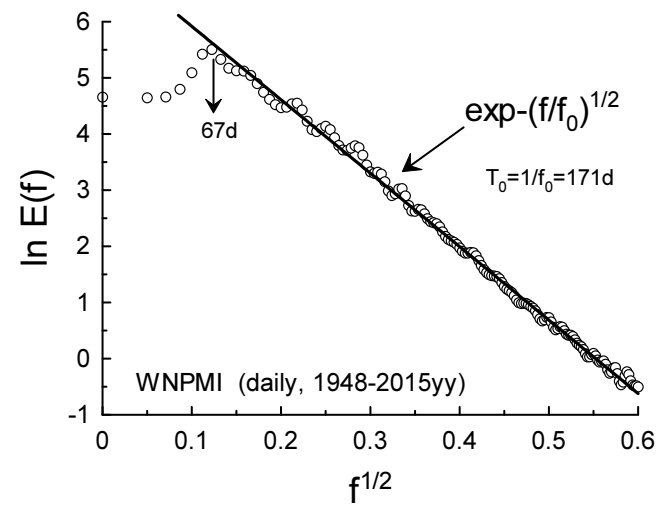

FIG. 14: Power spectrum for the intraseasonal daily WNPM index for the period 1948-2015yy (the data for the computation were taken from the site [43]).

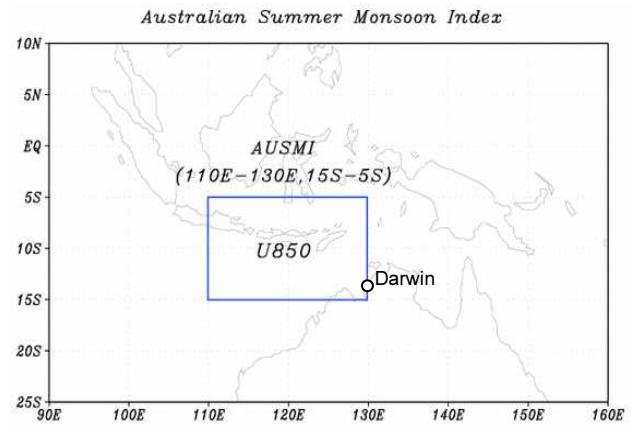

FIG. 15: The Australian Monsoon Index - AUSMI (adapted from the site [43]).

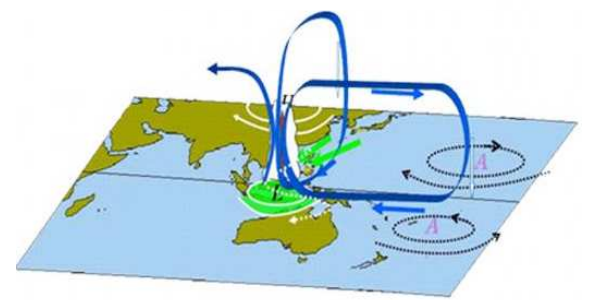

FIG. 16: A sketch of the broad-scale circulation corresponding to the active Australian Monsoon (adapted from the site [51]).

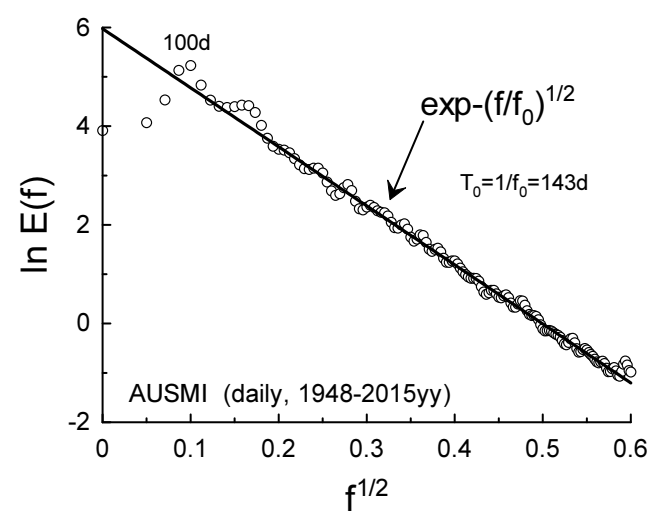

FIG. 17: Power spectrum for the intraseasonal daily AUSM index.

Despite this, convective activity related to the Asian Monsoon results in a global pattern expanding in both hemispheres for intraseasonal as well as for inter-annual time scales [45],[46]. Even Arctic ice patterns can be driven by the Asian Summer Monsoon via North Atlantic [47].

The intraseasonal time scales can be separated by removing of the components of interannual variability and annual cycle. Subtraction of a 120-day moving average is considered as an appropriate tool for removing the low frequency variations in such cases (see, for instance, Ref. [48] and references therein). We have used the 121-day moving average for this purpose:

$$
y(i)=\frac{\sum_{i-k}^{i+k} x(i)}{(2 k+1)}
$$

Figure 13 shows power spectrum for the intraseasonal (i.e. after the subtraction) daily ISM index for the period 1948-2015yy. The data for the computation were taken from the Ref. [43]. The straight line is drawn to indicate correspondence to the Eq. (3) with $\beta=3 / 4$. The characteristic time scale obtained from the best fit is $T_{0}=1 / f_{0} \simeq 32 d$. Figure 14 shows power spectrum for the intraseasonal daily WNPM index for the period 1948-2015yy (the data for the computation were taken from the site [43]). The straight line is drawn to indicate correspondence to the Eq. (3) now with $\beta=1 / 2$. The characteristic time scale obtained from the best fit is $T_{0}=1 / f_{0} \simeq 171 d$.

One can see that the both analytical types of the Hamiltonian distributed chaos are present in the Asian Monsoons. The above mentioned difference between its two components is reflected in the difference between the two values of the $\beta$ parameter (cf. Figs. 13 and 14). 


\section{Australian Monsoon}

The Australian Monsoon is a natural equatorial counterpart for the Asian (mainly WNPM) Monsoon. Indeed, flow across the Equator of the dry air from the continent, where a winter (for corresponding hemisphere) takes place, toward the hemisphere with summer conditions delivers moisture, that was taken on the way from the worm oceans. This moisture feeds the monsoon rains at the hemisphere with the summer conditions. The reverse of the winds direction each half a year results in the alternation between the monsoon rainfalls in the Northern and the Southern hemispheres.

The rainfall and wind records at the most northerly of the Australian capital cities - Darwin $\left(12^{\circ} \mathrm{S}, 130^{\circ} \mathrm{E}\right.$, see figure 15) were used to describe the Australian Monsoon in majority of early studies (see, for instance, the Refs. [49],[50] and references therein). However, as one can see from figure 16 a broad scale wind circulation index properly corresponding to the Australian Monsoon should be based at a different location. The authors of the recent paper Ref. [52] (see also an earlier paper Ref. [53]) suggested such index - AUSMI, based on $850 \mathrm{hPa}$ zonal wind anomalies averaged over the region $5^{\circ} \mathrm{S}-15^{\circ} \mathrm{S}, 110^{\circ} \mathrm{E}-130^{\circ} \mathrm{E}$ (see the Fig. 15). It should be noted that the Australian monsoon rainfall is located approximately in the area $\left(7.5^{\circ} \mathrm{S}-17.5^{\circ} \mathrm{S}, 120^{\circ} \mathrm{E}-150^{\circ} \mathrm{E}\right)$, i.e. well including the Darwin location. Despite this the AUSMI captures well not only the interannual and intraseasonal time scales variability of the Australian monsoonal rainfall but also the relationship of the Australian monsoon with the ENSO [52].

Figure 17 shows shows power spectrum corresponding to the intraseasonal daily AUSM index for the period 1948-2015yy (the data for the computations were taken from the site [43]). The straight line is drawn to indicate correspondence to the Eq. (2) with $\beta=1 / 2$. The characteristic time scale obtained from the best fit is $T_{0}=1 / f_{0} \simeq 143 d$. One can see that the AUSMI power spectrum corresponds to that of the WNPMI's type ( $\beta=1 / 2)$ rather than that of ISMI's type $(\beta=3 / 4)$. It could be expected, because the Australian monsoon has geographic setting in respect of the ocean-continent distribution more similar to that corresponding to WNPM rather than that corresponding to ISM.

\section{El Niño/La Niña}

El Niño and La Niña are opposite (the warm and cold correspondingly) phases of a chaotic cycle of the sea temperature fluctuations in the east-central Equatorial Pacific (see Fig.18 [54]). Southern Oscillation is the accompanying atmospheric component of the El Niño-Southern

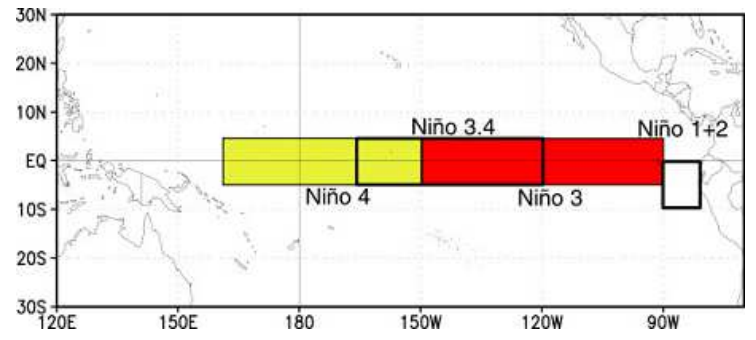

FIG. 18: Map of the tropical Pacific El Niño/La Niña regions.

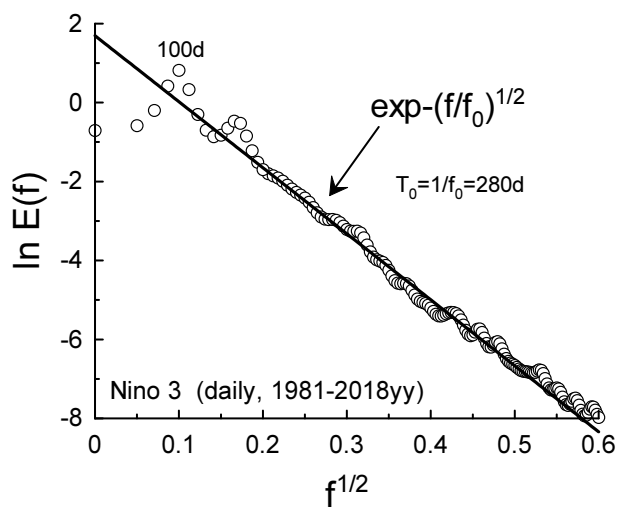

FIG. 19: Power spectrum for the intraseasonal daily Niño 3 index [60].

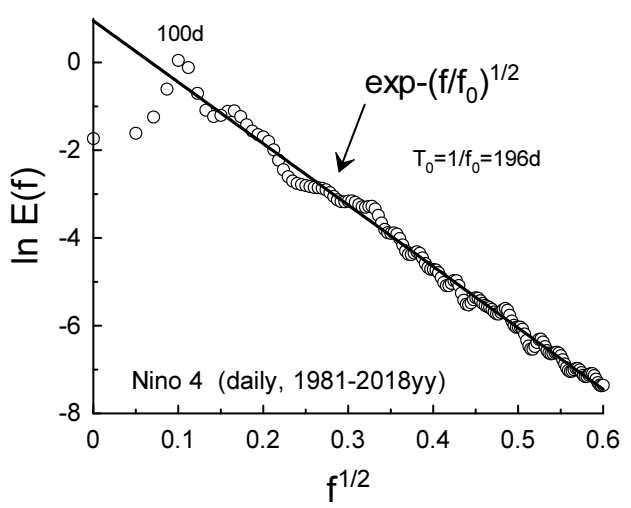

FIG. 20: Power spectrum for the intraseasonal daily Niño 4 index [60]. 
Oscillation (ENSO) pattern. The El Niño and La Niña phases result in high and low air surface pressure in the tropical western Pacific correspondingly.

For certain reasons these sea surface temperatures fluctuations and corresponding ocean-atmosphere interaction have large-scale impact on global climate.

Naturally relationship between the Asian Monsoons and El Niño/La Niña phenomenon is of great interest (see, for instance, Refs. [41],[55]-[59] and references therein). The relationships between the $\mathrm{El} \mathrm{Niño/La}$ Niña and the two subsystems of the Asian Monsoon are different, according to the difference between these subsystems. While the relationship between the El Niño/La Niña and the Indian Summer Monsoon is rather uncertain and non-stable the relationship between the El Niño/La Niña and the WNPM is much more strong and stable (cf. Fig. 12 and Fig. 18). Relationship between the Australian Monsoon and the ENSO is even stronger due to their geographic setting (cf. Figs. 15 and 18, and see below).

The Niño 3 (5N-5S, $150 \mathrm{~W}-90 \mathrm{~W}$ region) and the Niño 4 (5N-5S, 160E-150W region) indices, based on sea surface temperature (SST) anomalies averaged across a given region, can be considered as the relevant ones for comparison with the ISMI,WNPMI and AUSMI.

Figure 19 shows power spectrum computed for the Niño 3 intraseasonal (i.e after the subtraction of the 121 day moving average) daily index for 1981-2018yy period. The data for the computations were taken from site [60]). The straight line is drawn to indicate correspondence to the Eq. (3) with $\beta=1 / 2$. The characteristic time scale obtained from the best fit is $T_{0}=1 / f_{0} \simeq 280 d$. Figure 20 shows power spectrum computed for the Niño 4 intraseasonal daily index for 1981-2018yy period (the data for the computations were taken from site [60]). The straight line is drawn to indicate correspondence to the Eq. (3) with $\beta=1 / 2$. The characteristic time scale obtained from the best fit is $T_{0}=1 / f_{0} \simeq 196 d$.

Comparing the Figs. 13, 14 with the Figs. 19, 20 one can conclude that the Hamiltonian distributed chaos observed in the ENSO is more similar to the chaos observed in the WNPM than to that observed in the ISM. This conclusion is consistent with the other, above mentioned, observations. Moreover, compare the Fig. 17 with the Figs. 19 and 20 (cf. also Figs. 16 and 18) one can conclude that the Australian Monsoon belongs to an extended ENSO system (at least on the intraseasonal time scales).

\section{GLOBAL TEMPERATURE FLUCTUATIONS}

While dynamics of the global temperature long-term trends attracts major attention the statistical properties

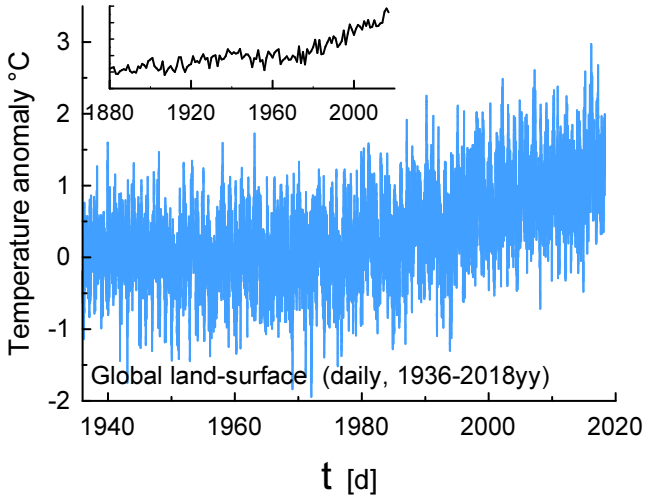

FIG. 21: Global average surface temperature anomaly for 1936-2018yy. The land-based daily data were taken from the Ref. [62]. The insert shows annual mean of the surface temperature anomaly (land-based) for 1880-2017yy.

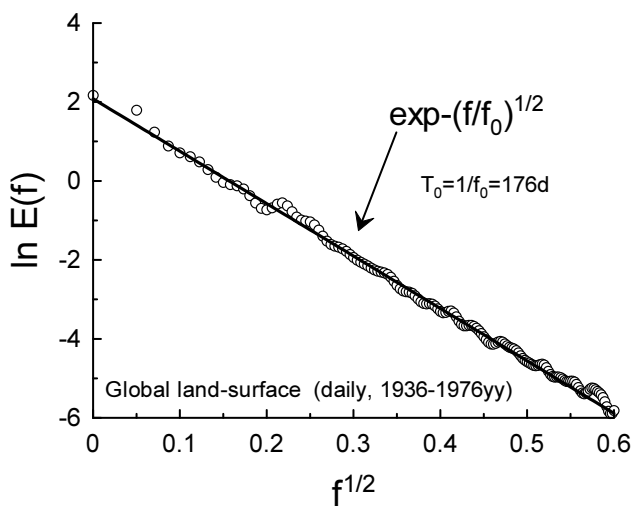

FIG. 22: Power spectrum corresponding to the sub-set (19361976yy) of the daily data shown in the Fig. 21.

of the global temperature fluctuations are much less studied. However, understanding of these properties are crucial both for analysis of the underlying physical processes and for a meaningful forecast. Recent developments in the historical temperature data processing resulted in appearance of the daily time series for a period of time sufficient for a statistical analysis [61],[62] (see Figure 21).

Figure 21 shows a global average surface temperature anomaly (relative to the Jan. 1951 - Dec. 1980 average). The land-based daily data for the period 1936-2018yy were taken from the site [62]. The entire daily time series shown in the Fig. 21 is statistically non-stationary and 


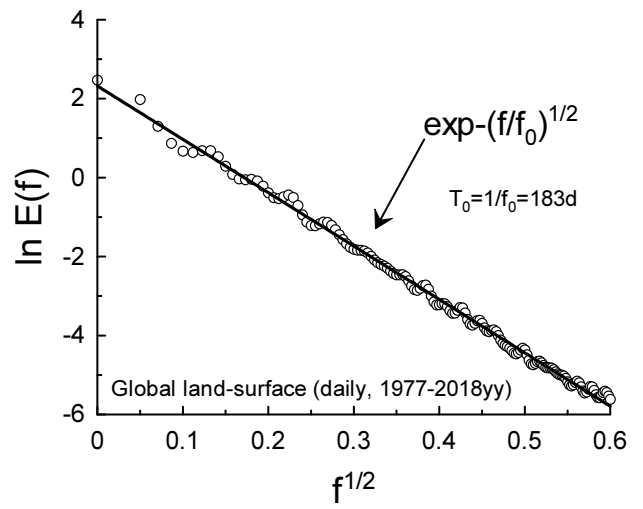

FIG. 23: Power spectrum corresponding to the sub-set (19772018 yy) of the daily data shown in the Fig. 21 (linear regression detrended).

a certain detrending is necessary before a spectral analysis. But before the detrending it should be noted that the time sub-series for the period 1936-1976yy is approximately statistically stationary. Figure 22 shows power spectrum corresponding to this statistically stationary sub-set of the data. The straight line in the figure (the best fit) indicates the stretched exponential decay Eq. (3) with $\beta=1 / 2$.

The second part of the time series, for the time period 1977-2018yy, can be detrended using the simplest linear regression method. Figure 23 shows corresponding power spectrum. The straight line in the figure (the best fit) indicates the stretched exponential decay Eq. (3) with $\beta=1 / 2$.

It is interesting that $T_{0}$ period is nearly a half-year (see Figs. 22 and 23). The global temperature fluctuations can be sensitive to this period due to dissimilarity between the Northern and Southern hemispheres geographic setting in respect of the land-ocean distribution. There is also more of a difference in solar radiation between winter and summer in the Southern hemisphere than in the Northern due to the Earth orbit eccentricity. The northward cross-equatorial ocean heat transport plays its role as well [63].

\section{PREDICTABILITY}

Let us define a smooth predictability (see Introduction). At smooth predictability the predictability horizon can be indefinitely extended by reducing the initial error.

At rough predictability the predictability horizon cannot be indefinitely extended by reducing the initial error. At this (rough) level of smoothness (less than $C^{\infty}$ ) the spectrum has a power-law form

$$
E(f) \propto f^{-\gamma}
$$

cf. the Refs. [7],[64] (it resembles the relation between smoothness and truncation of the Fourier transforms to finite time intervals).

The same level of smoothness - the $C^{\infty}$ class, that results in the stretched exponential spectrum Eq. (3) results also in the smooth predictability. Therefore, appearance of the stretched exponential spectrum Eq. (3) is a good indicator of the smooth predictability.

It should be also noted that for practical purposes the cases with $\beta=3 / 4$ have larger practical predictability horizons than those with $\beta=1 / 2$ (cf. Figs. 7, 10, 13, with Figs. 5,11,14).

\section{ACKNOWLEDGEMENT}

I gratefully acknowledge use of the data provided by the Zenodo database (CERN), the Earth system research laboratory (NOAA), the KNMI Climate Explorer (Netherlands), the Asia-Pacific Data Research Center at the University of Hawaii and the Berkeley Earth dataset (USA). I also thank B. Galperin and A. Pikovsky for discussions.

[1] E.N. Lorenz, J. Atmos. Sci., 20, 130 (1963).

[2] N. Ohtomo, K. Tokiwano, Y. Tanaka et. al., J. Phys. Soc. Jpn. 641104 (1995).

[3] J. D. Farmer, Physica D, 4, 366 (1982).

[4] D.E. Sigeti, Phys. Rev. E, 52, 2443 (1995).

[5] A. Bershadskii, EPL, 88, 60004 (2009).

[6] S.M. Osprey and M.H.P Ambaum, Geophys. Res. Lett. 38, L15702 (2011).

[7] E.N. Lorenz, Tellus, XXI (3), 289 (1969).

[8] A. Bershadskii, arXiv:1805.06820; arXiv:1803.10139 (2018).

[9] T.G. Shepherd, Advances in Geophysics, 32, 287 (1990).

[10] T.G.Shepherd, Encyclopedia of Atmospheric Sciences, J. R. Holton et al., Ed., 929 (Academic Press, 2003).

[11] V. Pelino et al., Commun. Nonlinear Sci. Numer. Simulat., 17, 2122 (2012).

[12] A. Gluhovsky, and K. Grady, Chaos, 26, 023119 (2016).

[13] R.Z. Sagdeev, D.A. Usikov, G.M. Zaslavsky, Nonlinear Physics: from the Pendulum to Turbulence and Chaos (Harwood, New York, 1988).

[14] D.C. Johnston, Phys. Rev. B, 74, 184430 (2006).

[15] S. Ashkenazi and V. Steinberg, Phys. Rev. Lett. 83, 3641 (1999).

[16] C. Tong and A. Gluhovsky, Phys. Rev. E 65, 046306 (2002).

[17] A. Gluhovsky, Nonlinear Processes in Geophysics, 13, 125 (2006).

[18] X-Z. Wu, L. Kadanoff, A. Libchaber, and M. Sano, Phys. Rev. Lett. 64, 2140 (1990). 
[19] J.C. Sprott, Chaos and Time-Series Analysis (Oxford. University Press, 2003).

[20] http://sprott.physics.wisc.edu/cdg.htm

[21] J.W. Hurrell, Science, 269, 676 (1995).

[22] P.D. Jones, T. Jonsson and D. Wheeler, Int. J. Clim., 17, 1433 (1997).

[23] T. Cropper et al., Geosci. Data J., 2, 12 (2015).

[24] D.W.J. Thompson and J.M. Wallace, Res. Lett. Geophys., 25, 1297 (1998).

[25] D.W.J. Thompson and J.M. Wallace, J. Clim., 13, 1000 (2000).

[26] C.K Folland et al., J. Clim., 22, 1082 (2009).

[27] T. Cropper et al., http://doi.org/10.5281/zenodo.9979

[28] J.W. Hurrell et al., in The North Atlantic Oscillation: Climatic Significance and Environmental Impact, Geophysical Monograph 134, p.1, American Geophysical Union (2003).

[29] https://www.esrl.noaa.gov/psd/data/timeseries/daily/PNA

[30] E. Kalnay et al., Bull. Amer. Meteor. Soc., 77, 437 (1996).

[31] V. Magana, J. Geophys. Res., 98, 10441 (1993).

[32] S.L. Marcus, M. Ghil, and J.O. Dickey, J. Atmos. Sci., 51, 1431 (1994).

[33] A.W. Robertson, and C.R. Mechoso, Mon. Wea. Rev., 131, 1566 (2003).

[34] Ch.A.C. Cunningham and I.F. De Albuquerque Cavalcanti. Int. J. Climatology, 26, 1165 (2006).

[35] A. Bershadskii, arXiv:1806.01750 (2018).

[36] J.M Wallace, On the role of the Arctic and Antarctic oscillations in polar climate. ECMWF Seminar on Polar Meteorology (2006). Available at the site: https://www.ecmwf.int

[37] M.P. Baldwin and T.J. Dunkerton, J. Geophys. Res., 104, 30937 (1999).

[38] http://www.cpc.ncep.noaa.gov/products/precip/CWlink

[39] https://www.esrl.noaa.gov/psd/data/20thC_Rean/ timeseries/daily/AO/

[40] https://www.esrl.noaa.gov/psd/data/20thC_Rean/ timeseries/daily/AAO/
[41] B. Wang, R. Wu and K.-M. Lau, J. Climate, 14, 4073 (2001).

[42] B. Wang and Z. Fan, Bull. Amer. Meteor. Soc., 80, 629 (1999).

[43] http://apdrc.soest.hawaii.edu/projects/monsoon/

[44] A. Bershadskii, Phil. Trans. R. Soc. A, 371, 20120168 (2013).

[45] H. Lin, J. Atmos. Sci. 66, 2697 (2009).

[46] Q. Ding, Q et al., 2011. J. Climate, 24, 1878 (2011).

[47] G. Grunseich and B. Wang, J. Climate, 29, 9097 (2016).

[48] M.J. Ventrice et al., Monthly Weather Review, 141, 4197 (2013).

[49] K.Y. Kim et al., J. Geophys. Res., 111, D20105 (2006).

[50] R. Suppiah, International Journal of Climatology, 24, 269 (2004).

[51] http://www.clivar.org/asian-australian-monsoon

[52] Y. Kajikawa, B. Wang and J. Yang, International Journal of Climatology, 30, 1114 (2010).

[53] B. Wang, I.S. Kang and J.Y. Lee, Journal of Climate, 17, 803 (2004).

[54] http://www.cpc.ncep.noaa.gov/products/analysis_monitoring /ensostuff/nino_regions.shtml

[55] V. Krishnamurthy and B. N. Goswami, J. Climate, 13, 579 (2000).

[56] B. Wang et al., Geophys. Res. Lett., 32, L15711 (2005).

[57] C. Chou, J.Y. Tu and J.Y. Yu, J. Climate, 16, 22752287 (2003),

[58] W. Jiang et al., J. Climate, 30, 109 (2017).

[59] J. Crétat et al., Climate Dynamics, 49, 1429 (2017).

[60] https://climexp.knmi.nl/start.cgi

[61] R. Rohde et al., Berkeley Earth Temperature Averaging Process. Geoinfor Geostat: An Overview 1:2. doi:10.4172/gigs.1000103 (2013).

[62] http://berkeleyearth.lbl.gov/auto/Global/

[63] S.M. Kang et al., Climate Dynamics, 44, 1457 (2015).

[64] T.Y. Leung, et al., Geophys. Res. Abstracts, 20, EGU2018-371 (2018). 